\title{
Duty Cycle and Channel Occupancy Rate Estimation with MED-FCME LAD ACC
}

\author{
Janne Lehtomäki*, Risto Vuohtoniemi* and Kenta Umebayashi ${ }^{\dagger}$ \\ ${ }^{*}$ Centre for Wireless Communications (CWC) \\ University of Oulu, Oulu, Finland \\ Email: jannel@ee.oulu.fi \\ ${ }^{\dagger}$ Department of Electrical and Computer Engineering \\ University of Agriculture and Technology, Tokyo, Japan \\ Email: ume_k@cc.tuat.ac.jp
}

\begin{abstract}
Duty cycle (DC) and channel occupancy rate (COR) measurements are important to characterize the availability of white space for cognitive radio systems. To facilitate accurate DC measurements, we apply the localization algorithm based on double-thresholding (LAD) with the adjacent cluster combining (ACC). In order to get stable thresholds, we propose and apply a modified version of the LAD ACC called the MED-FCME LAD ACC. We show that LAD ACC based approaches can also be used for COR estimation. For even more accurate COR estimation, we propose estimation based on hard decision fusion of the frequency domain decisions. Measurement results confirm the good performance of the proposed approaches.
\end{abstract}

\section{INTRODUCTION}

Spectrum measurements are important to characterize and model the availability of white space for cognitive radio systems (CRS). Most of the previous spectrum measurements have been power measurements and the classification into signal (1) or noise (0) has been done with a threshold $\gamma[1]$, [2]. In these measurements, the frequency resolution is very accurate (in the order of $10-300 \mathrm{kHz}$ ) so they are suitable for all kinds of signals. The total measurement is divided into time intervals. The duty cycle (DC) (0-100\%) for each frequency bin and given time interval is the fraction of $1 \mathrm{~s}$ in the corresponding elements of the binary matrix $D$.

The DC calculation (if performed real time) can be viewed to be a pre-processing which enables long measurements, since it can be difficult to record the raw data (power measurement values) over a large time period due to the data storage and bandwidth limitations. However, by using compression for a bit-packed data format it is also possible to record the full binary matrix $D$ [3] with more than 50 billion $\left(50 \cdot 10^{9}\right)$ data points [4].

Due to frequency selective channel fading and receiver noise, the sensitivity of the classification is reduced. To address this problem, the localization algorithm based on double-thresholding (LAD) with the adjacent cluster combining (ACC) uses two thresholds, upper and lower [5]. Signals are detected according to the lower threshold thus improving sensitivity. The problem of too many false alarms is avoided

The research leading to these results was derived from the European Community's Seventh Framework Programme (FP7) under Grant Agreement number 248454 (QoSMOS). by requiring that all contiguous groups of detected signals should have at least one sample also exceeding the stricter upper threshold. In order to further avoid incorrectly classifying signal samples as only noise, in the ACC processing contiguous groups that are separated by $n$ or less samples are joined and gaps between them are filled.

In channel occupancy rate (COR) measurements the purpose is to measure the availability of a channel(s) [4], [6], [7]. Usually in spectrum use measurements, the average DC is considered [1], [2]. However, the average DC is not necessarily sufficient to characterize the availability of channels for a CRS. For example, consider the case of a narrowband signal (such as a wireless microphone) within a wideband channel (such as a television channel). Since the CRS should protect co-existing users even if narrowband, the average DC is not giving the proper picture. Maximum based combining of DC values was recommended for COR estimation in [4]. The COR estimation can be also used to adapt CRS operating parameters to the current environment [6].

The verification of the availability of spectrum white space for CRSs needs to be flexible regarding channel bandwidths and center frequencies. A typical approach to detect if a signal is present in a channel or not is to compare channel power measurements to a threshold (energy detector $\mathrm{ED} /$ radiometer) [1], [8]. This approach has a good performance for detecting unknown signals, with some drawbacks such as that it requires typically fixed channels. The maximum combining is based on the DC data thus it is suitable for long measurement durations. It can be performed after measurement (post-processing) in which case there is no need to fix the studied channels and/or during measurement.

In this paper, we propose and apply a modified version of the LAD ACC called the MED-FCME LAD ACC for DC estimation and for COR estimation. The LAD ACC has not previously been applied to DC/COR estimation. The proposed approach is fully flexible regarding channel bandwidths and center frequencies. We also propose a new technique for even more accurate COR estimation that is based on the availability of the full binary matrix $D$. 


\section{MeAsurement CONFIGURATION}

The measurement configuration is summarized in Table I. The studied band was the 2400-2500 MHz ISM band. The $2.4 \mathrm{GHz}$ ISM band is a very important band worldwide so cognitive techniques helping smooth operation on that band would be highly important. Already, some commercial systems use spectrum monitoring for self-healing and self-optimizing wireless network in $2.4 \mathrm{GHz}$ ISM band ${ }^{1}$. The bandwidths of the typical signals in $2.4 \mathrm{GHz}$ ISM band vary from $1 \mathrm{MHz}$ (Bluetooth) to more than $20 \mathrm{MHz}$ (802.11).

The number of frequency points was $N=916$. The sweep time was $\sim 10 \mathrm{~ms}$. This sweeping is fast compared with previous studies based on typical spectrum analyzers [2]. The sweeping process is repeated continuously. For example one hour measurement corresponds to around 360000 sweeps and more than $3 \cdot 10^{7}$ data points. A record is defined to correspond to $T=200$ complete sweeps so that one hour corresponds to around 1800 records. We denote the received frequency domain power data with $P_{i, c}^{k}$, where $k$ is the record index, $c=1,2, \cdots, N$ is the frequency bin index and $i$ is the sweep index within the record $(i=1,2, \cdots, T)$. The instrument is based on fast Fourier transforms (FFTs). The time required to collect the samples needed by the FFT was around 10 $\mu \mathrm{s}$ for a frequency segment. This is comparable to $802.11 \mathrm{~b}$ clear channel assessment $(\mathrm{CCA})$ duration $(\leq 15 \mu$ s $[9$, Sect. 15.3.3]).

TABLE I

MEASUREMENT CONFIGURATION

\begin{tabular}{ll}
\hline Instrument & Agilent N6841A RF sensor \\
Center frequency & $f_{c}=2450 \mathrm{MHz}$ \\
Frequency span & $100 \mathrm{MHz}$ \\
Resolution bandwidth & $242.27 \mathrm{kHz}$ \\
Frequency bin separation & $109.3750 \mathrm{kHz}$ \\
Window type & Gausstop window \\
Number of frequency points & $N=916$ \\
Sweep time & $\sim 10 \mathrm{~ms}$ \\
Average type & Off \\
\hline Antenna & Linksys ISM band dipole antenna \\
Filter & Creowave ISM band filter \\
Low noise amplifier & Mini-Circuits ZRL-3500 \\
\hline FCME parameter & $T$ CME $=4.6052$ \\
MED-FCME filtering length & $M=800$ (around 8 seconds) \\
ACC parameter & $n=8$ \\
Record duration & $T=200$ sweeps $(\sim 2$ s) \\
\hline Thresholds: & \\
MED-FCME & 10 dB above adaptive noise floor \\
Upper (MED-FCME LAD ACC) & 10 dB above adaptive noise floor \\
Lower (MED-FCME LAD ACC) & 6 dB above adaptive noise floor \\
\hline Signal generation & Agilent E4438C ESG \\
Radio channel emulator & + Agilent Signal Studio software \\
\hline
\end{tabular}

\section{NOISE FLOOR ESTIMATION}

In conventional single threshold detection, the received power levels $P_{i, c}^{k}$ (the raw received data) are compared with

\footnotetext{
${ }^{1}$ http://www.cisco.com/en/US/netsol/ns1070/index.html
}

detection thresholds $\gamma_{i, c}^{k}$. If a received power level exceed the threshold, we declare that a signal was present. Otherwise, we declare that only noise was present. Thus [1],

$$
D_{i, c}^{k}= \begin{cases}1, & P_{i, c}^{k}>\gamma_{i, c}^{k} \\ 0, & \text { otherwise }\end{cases}
$$

where $D_{i, c}^{k}$ are the binary decisions made about the signal presence or absence.

Usually, the threshold is set so that the probability of only noise causing the detection threshold to be exceeded (the probability of false alarm, $\left.P_{\mathrm{FA}}\right)$ is controlled. Let us denote the target false alarm probability with $P_{\mathrm{FA}}$. If we know the distribution of the noise, we can use a threshold that is $m$ $\mathrm{dB}$ above the noise floor (NF). Since windowed magnitudesquared FFT data follows the exponential distribution [10], we can calculate the proper $m$ as $m=10 \log _{10}\left(-\log \left(P_{\mathrm{FA}}\right)\right)$ [dB]. A significant issue is the uncertainty about the NF. Additionally, the NF can change as a function of time [11]. Thus, the NF estimate should be updated regularly.

Assuming a sufficiently static situation, the correct threshold for each frequency bin can be found out by using the PFA method proposed by López-Benítez in [1]. In this method, we collect a sufficient number of samples that are guaranteed to be noise-only. Then we simply find out the detection threshold that is exceeded with probability equal to the desired false alarm probability $\dot{P}_{\mathrm{FA}}$. This threshold is independent of time but varies with the frequency bin index.

We apply the MED-FCME [4], [12] for estimating the NF. The MED-FCME estimates the NF directly from the signal (with the antenna connected) while simultaneously performing regular measurements. The MED-FCME does not require dedicated time periods during or before the measurements to estimate the noise floor. In each sweep, the MED-FCME uses the forward consecutive mean excision (FCME) algorithm [13] for deciding which frequency bins are clean (do not contain signals). The sensitivity of the FCME is controlled by the FCME parameter $T_{\mathrm{CME}}$. The $\mathrm{NF}$ is estimated from the assumed clean frequency bins. The NF estimates by the FCME are filtered with a median filter of length $M$ leading to the MED-FCME threshold $\gamma_{i, c}^{k}=\hat{N F_{i}^{k}}+m$, where $\hat{N F_{i}^{k}}$ is the MED-FCME NF estimate in $\mathrm{dBm}$.

It has been shown that the MED-FCME algorithm leads to much improved threshold accuracy as compared to the baseline FCME algorithm [12]. The benefit of reduced variance due to filtering is obvious. Although the FCME is a significantly robust algorithm, it can catastrophically fail when a large portion of the band contains signals, temporarily giving up to $15 \mathrm{~dB}$ too large thresholds [12]. However, the median will not fail when some of the FCME NF estimates it is based on are incorrect. Also, the median is much more robust as compared to, for example, the mean. Another benefit is that the MED-FCME protects against improper $T_{\mathrm{CME}}$.

Theoretically the proper $m$ for a given target false alarm probability $P_{\mathrm{FA}}$ for MED-FCME can be derived based on the results in [4] as 


$$
m=10 \log _{10}\left(\frac{-\log \left(\dot{P_{\mathrm{FA}}}\right)}{\sum_{j=1}^{N-\hbar} \frac{(N-\hbar-j+1)}{(N+1-j)(N-\hbar)}}\right),
$$

where $\hbar=\lceil\operatorname{CSRR} \times N\rceil$, where $\mathrm{CSSR}=e^{-\mathrm{TCME}}$ or from [14]. The equation (2) includes a correction factor for taking into account the clean samples incorrectly classified as signals by the FCME.

In order to compare the MED-FCME thresholds with the PFA method thresholds we performed a measurement in an anechoic chamber in order to guarantee strictly noise-only samples. The filter input was terminated using $50 \Omega$ instead of an antenna. Fig. 1 shows the comparison of the MED-FCME thresholds with the PFA method thresholds derived with target $\dot{P_{\mathrm{FA}}}=10^{-4}$ and $\dot{P_{\mathrm{FA}}}=0.03$. The theoretical values for $m$ corresponding to these target false alarm probabilities are 9.9 $\mathrm{dB}$ and $5.7 \mathrm{~dB}$, respectively. However, we used slightly higher values of $10 \mathrm{~dB}$ and $6 \mathrm{~dB}$ to protect against the non flat NF. The measurement duration was one hour and the PFA method threshold was set based on all received data during the hour. Since the MED-FCME threshold varies with time, the figure shows the range of thresholds given by the MED-FCME from the minimum to maximum during the hour. It can be seen that MED-FCME thresholds were within $\pm 1 \mathrm{~dB}$ of the values given by the PFA method and usually the difference was much less than this. The situation was mostly static. In dynamic cases with changing NF the proposed method is expected outperform the PFA method, depending on how much the NF is changing.

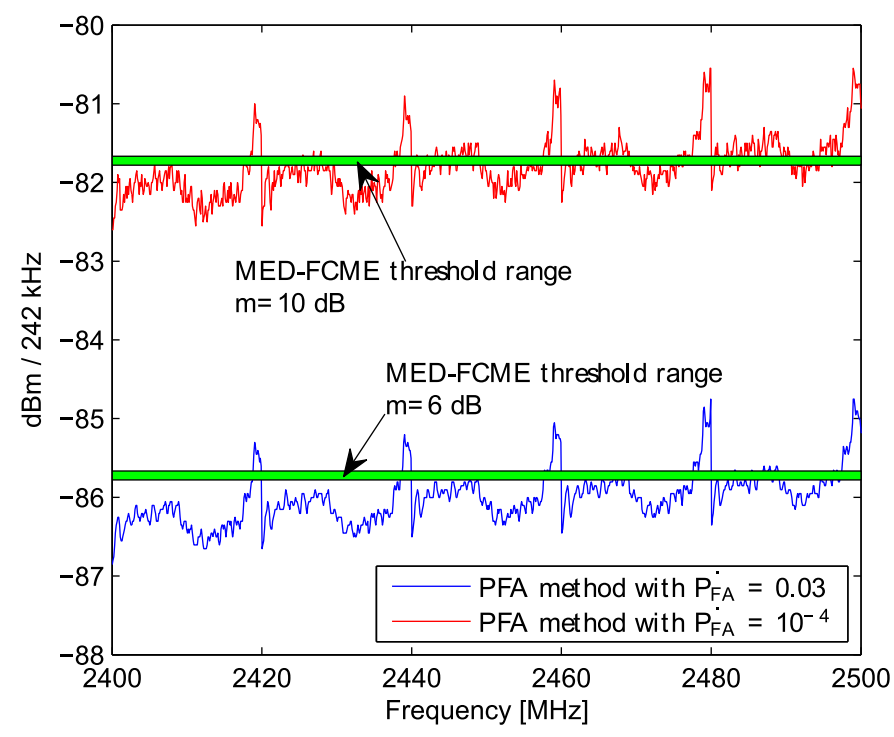

Fig. 1. Comparison of MED-FCME thresholds to PFA method thresholds (external gain around $\sim 20 \mathrm{~dB}$ ).

We performed one week measurement in the Oulu city center in June 2011. We may estimate the actual probability of false alarm and thus the threshold setting accuracy from the results in the frequency range $2483.5-2500 \mathrm{MHz}$ that is allocated to satellite downlink and thus has a very low utilization indoors. Results in Fig. 2 using $m=10 \mathrm{~dB}$ $\left(\dot{P_{\mathrm{FA}}}=10^{-4}\right)$ indicate sufficiently accurate threshold setting also in an actual radio environment.

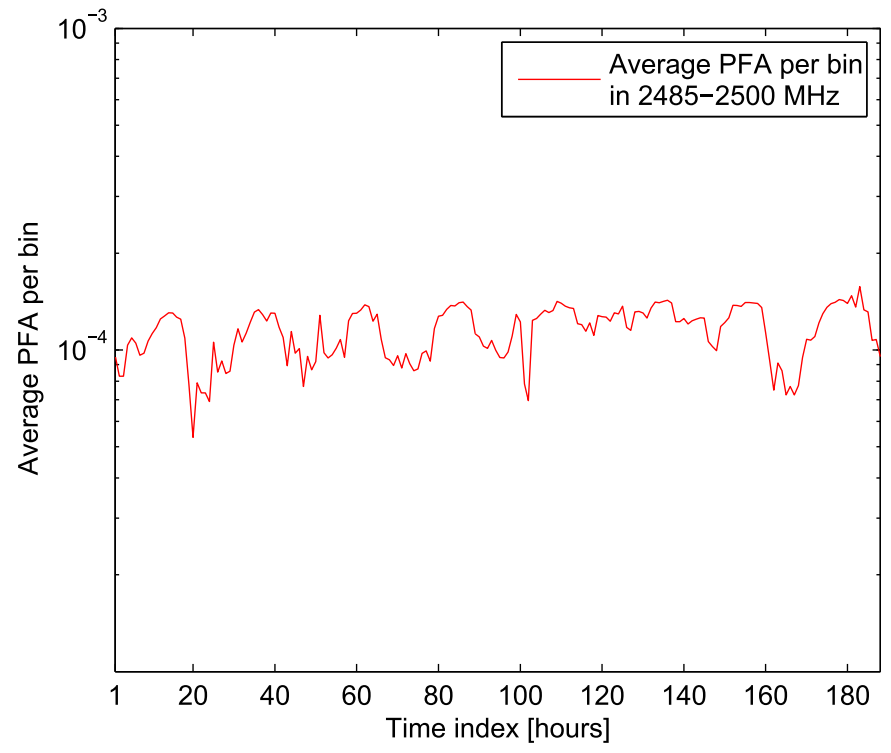

Fig. 2. Probability of false alarm per frequency bin with MED-FCME algorithm. Measurement performed in the Oulu city center in June 2011, target $P_{\mathrm{FA}}^{\cdot}=10^{-4}$.

\section{DC ESTIMATION}

For each individual frequency bin $c$, a DC estimate $S_{c}^{k}$ based on the binary spectral occupancy matrix for record $k$ is [1], [2]

$$
S_{c}^{k}=\frac{\sum_{i=1}^{T} D_{i, c}^{k}}{T}
$$

\section{A. MED-FCME}

The case where the DC estimates are based on binary spectrum occupancy matrix found with a single threshold detection (1) with threshold derived using the adaptive MEDFCME NF estimates is called simply "MED-FCME". The MED-FCME was proposed in [4].

\section{B. MED-FCME LAD ACC}

We apply the LAD ACC for DC measurements. This is done by using LAD ACC for calculating $D_{i, c}^{k}$ and using (3). The LAD method applies adaptive thresholding [15]. The conventional LAD ACC uses the FCME algorithm [13] for threshold setting. In the modified version proposed here called MED-FCME LAD ACC, the upper and lower thresholds are derived using the NF estimated by the MED-FCME algorithm [4], [12] for much improved threshold stability.

Fig. 3 shows an example of the measurement process. The signal was IEEE $802.11 \mathrm{~b}$ with center frequency $2472 \mathrm{MHz}$ and the reference duty cycle (the actual/correct DC) was $40 \%$. The subplot a) shows the measured DC estimates $S_{c}^{k}$ by the 
MED-FCME for frequency bins between 2450-2490 MHz (the measurement actually covered the full ISM band) and for record indices $k=1,2, \cdots, 43$. The subplot c) shows the 43 traces of $S_{c}^{k}$ plotted in the same figure, all with red color. The subplots b) and d) show the same results by the MED-FCME LAD ACC. This result confirms benefits of the MED-FCME LAD ACC for DC measurements. Additionally, the results are much more visually pleasing than with MEDFCME. These gains are due to the lower threshold and also due to the ACC processing.
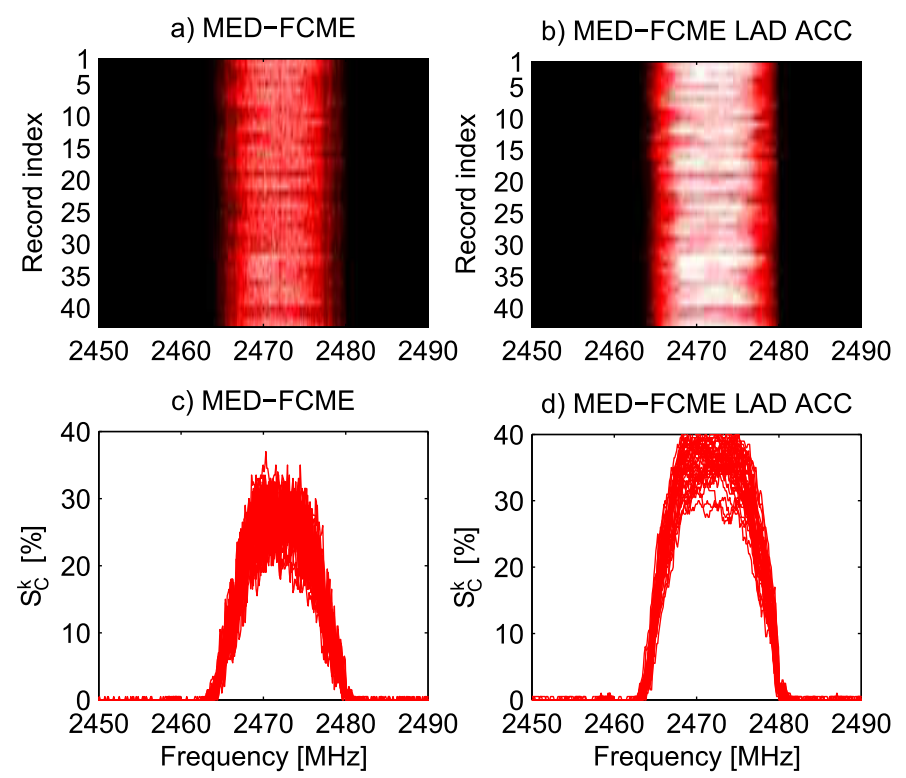

Fig. 3. Measument results $\left(S_{c}^{k}\right), 802.11 \mathrm{~b}$ signal, ETSI BRAN WLAN channel model A, device speed $5 \mathrm{~km} / \mathrm{h}$, channel emulator output level $-76 \mathrm{dBm}$.

\section{COR ESTIMATION}

\section{A. Maximum processing of DC estimates $S_{c}^{k}$}

It was shown in [4] that maximum combining of the DC estimates $S_{c}^{k}$ is suitable for COR estimation. The maximum will classify the channel as occupied even if only a part of it is actually occupied. This is intuitive since a CRS should protect co-existing users even if narrow band, using different channelization, from adjacent overlapping channels, etc. Formally, the COR estimate for a CRS channel $s$ with the maximum processing is [4]

$$
L_{s}^{k}=\max _{c \in \Theta_{s}} S_{c}^{k}=\max _{c \in \Theta_{s}} \frac{\sum_{i=1}^{T} D_{i, c}^{k}}{T}
$$

where $\Theta_{s}$ is the set of frequency bins belonging to the CRS channel $s$.

In principle, there is no limitation on the CRS channels and they can overlap with each other such as in 802.11 and they can have variable bandwidths.

\section{B. r-out-of- $\left|\Theta_{s}\right|$ hard decision fusion}

Hard decision fusion has been widely studied in cooperative spectrum sensing for combining individual local decisions in order to form one fused detection decision. We propose hard decision fusion with the " $k$-out-of- $N$ " fusion rule for the individual binary sensing decisions of a sensor within a CRS channel $s$. We denote our approach $r$-out-of- $\left|\Theta_{s}\right|$. The fused decision for sweep $i$ within a record $k$ is $\Lambda_{i, s}^{k}=1$ if $\sum_{c \in \Theta_{s}} D_{i, c}^{k} \geq r$ and $\Lambda_{i, s}^{k}=0$ otherwise. The corresponding COR estimate for the CRS channel $s$ in record $k$ is given by

$$
L_{s}^{k}=\frac{\sum_{i=1}^{T} \Lambda_{i, s}^{k}}{T}
$$

The binary decisions $D_{i, c}^{k}$ are assumed to be made using the MED-FCME. The reason for this is that if the MED-FCME LAD ACC has at least one 1, the MED-FCME must also have at least one 1. This is because the upper threshold of the MEDFCME LAD ACC is equal to the MED-FCME threshold.

We point out that Hamming weight (number of 1's) of hardquantized FFT amplitudes has previously been utilized in [16] by Levitt et al. although for significantly different strategy.

\section{Energy detector (ED)}

In the ED, we measure the channel power from the whole channel and compare it to a threshold. Geirhofer et al. have used the ED for modeling 802.11 signals [17]. Usually energy detection is performed in time domain by summing magnitude-squared received samples. The noise variance for $\mathrm{ED}$ is reduced due to the utilization of several samples (time-bandwidth product). This allows the use of a lower threshold. Channel power can also be measured by summing the frequency bin power samples ${ }^{2}$ corresponding to the CRS channel $s$. This is the approach utilized herein so that the ED decision for sweep $i$ within a record $k$ is $\Lambda_{i, s}^{k}=1$ if $\sum_{c \in \Theta_{s}} P_{i, c}^{k} \geq \alpha$, and $\Lambda_{i, s}^{k}=0$ otherwise. The $L_{s}^{k}$ is found as in (5). An interesting point is that the ED can be viewed to be soft decision fusion as opposed to hard decision fusion utilized in the $r$-out-of- $\left|\Theta_{s}\right|$. Since we derived the ED thresholds $\alpha$ with the PFA method we did not have to consider calibration issues for accurate channel power estimation.

\section{MEASUREMENT SETUP}

The signal was generated with Agilent E4438C ESG Vector Signal Generator and the Agilent Signal Studio software for WLAN. The channel emulator used was state-of-the-art Elektrobit (EB) Propsim F8. The reference COR 40\% was obtained by controlling the idle time between packets. The reference occupancy level was equal to the true time-domain occupancy level that can be defined to be the fraction of the measurement time with the signal present, with infinite time resolution. The signal was transmitted using a short cable to the channel emulator input. The channel emulator output was connected to receiver input of the energy detection system. We considered both the additive white Gaussian noise (AWGN) channel and a more realistic ETSI BRAN WLAN channel model A. The model A aims to model a typical office environment with NLOS conditions [18].

\footnotetext{
${ }^{2}$ Agilent Spectrum and Signal Analyzer Measurements and Noise, Application Note
} 


\section{MEAsurement RESUlts}

In most of the measurements the co-existing user's signal was IEEE $802.11 \mathrm{~b}$ with $11 \mathrm{Mbit} / \mathrm{sec}$ data rate, total packet size 1508 bytes, and center frequency $2472 \mathrm{MHz}$ (channel 13). We estimated the COR of different CRS channels and compared it to the true value $(40 \%)$. We studied both $2 \mathrm{MHz}$ IEEE 802.15.4 channels and 15 MHz CRS channel corresponding one possible component carrier bandwidth of the LTE-A. The 802.15.4 standard is suitable for low-rate wireless personal area networks and it is vulnerable to interference from 802.11.

\section{A. 802.15.4 channels}

Experimental validations with a real 802.15.4 node have shown that its CCA cannot sense $802.11 \mathrm{~b}$ signals when $802.11 \mathrm{~b}$ signal power is $-81 \mathrm{dBm}$ or less [19, Yuan2010]. They studied a 802.15.4 channel with center frequency having $2 \mathrm{MHz}$ offset to the $802.11 \mathrm{~b}$ signal center frequency. Fig. 4 shows measurement resuls for AWGN channel. The coexisting signal was $802.11 \mathrm{~b}$. It can be seen that the MEDFCME LAD ACC has a sufficient performance as compared to a real 802.15.4 node. The frequency domain hard decision fusion had somewhat better performance and the ED had the best performance. However, the differences are not radical and all three methods were within $5 \mathrm{~dB}$ and are significantly better than conventional single threshold detection (MED-FCME). It can be seen that the performance reduces the larger the offset is. Fig. 5 shows the performance for the channel model A. It can be seen that performance is generally decreased due to fading. However, with very low signal power values fading does actually help a little bit. The reason is that fading can be both destructive and constructive and sometimes without constructive fading detection is not possible.

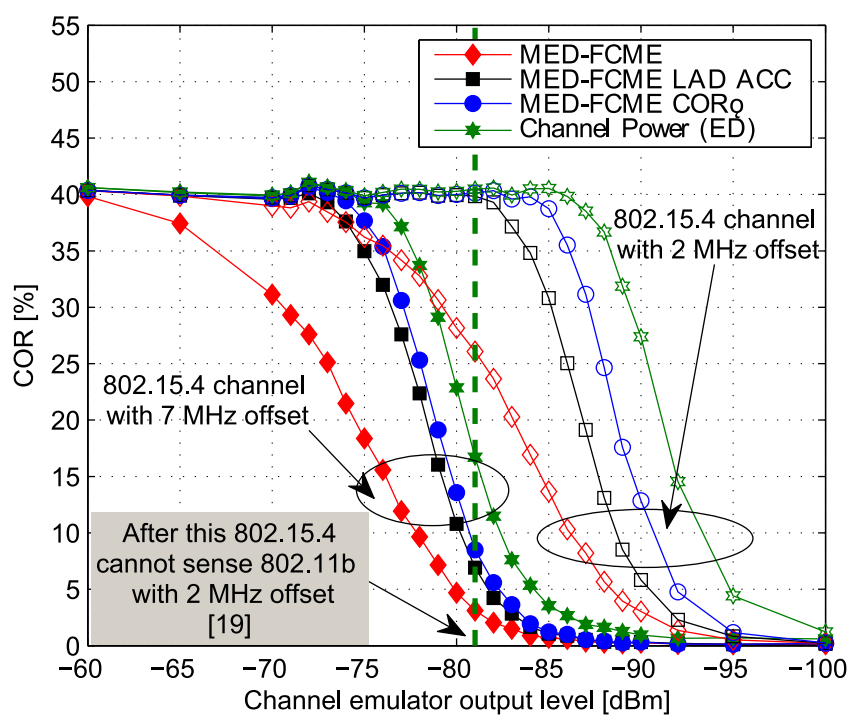

Fig. 4. Measurement results $\left(L_{s}^{k}\right), 802.11 \mathrm{~b}$ signal, AWGN channel, $r=1$.

\section{B. $15 \mathrm{MHz}$ CRS channel}

Figs. 6-7 show measurement results with a $802.11 \mathrm{~b}$ signal. The COR results are shown for a CRS channel with center frequency $2472 \mathrm{MHz}$ and $15 \mathrm{MHz}$ bandwidth. For reference, a energy detection threshold specified in the IEEE 802.11 standard for CCA is $-76 \mathrm{dBm}$ [9, Sect. 15.4.8.4]. Again, it can be seen the MED-FCME LAD ACC has a sufficient performance since performance was exceeding or equal to standard requirements. This is a remarkable result since the MED-FCME LAD ACC preserves the full frequency resolution. It can also be seen that frequency domain hard decision fusion and ED had even better performance.

Fig. 8 shows measurement results with a wideband FM signal (many wireless microphones use the FM modulation). The signal ways always on, so that the reference COR is $100 \%$. The bandwidth was around $400 \mathrm{kHz}$. It can be seen that the MED-FCME LAD ACC had the best performance and the ED had the worst performance. The reason is that the FM signal can be buried under the noise from the whole CRS channel.

\section{CONCLUSIONS}

We proposed and evaluated a system and methodologies for DC and COR measurements. The proposed approaches are generic and not limited to a specific band and signal structure. Measurement results showed that the MED-FCME LAD ACC can be used for accurate DC estimation. The generic MED-FCME LAD ACC approach showed also sufficient performance for COR estimation. Measurement results showed that the proposed frequency domain hard decision fusion is suitable for even more accurate COR estimation with less storage requirements and complexity than the ED. In fact, there is no reason why both of the approaches cannot be used simultaneously so that MED-FCME LAD ACC is used for accurate DC estimation and the hard decision fusion is used for accurate COR estimation.

\section{REFERENCES}

[1] M. López-Benítez and F. Casadevall, "Methodological aspects of spectrum occupancy evaluation in the context of cognitive radio," Eur. Trans. Telecomm., vol. 21, no. 8, pp. 680-693, Dec. 2010.

[2] M. Wellens, "Empirical modelling of spectrum use and evaluation of adaptive spectrum sensing in dynamic spectrum access networks," $\mathrm{Ph} . \mathrm{D}$. dissertation, RWTH Aachen University, Germany, May 2010.

[3] J. Naganawa, K. Hojun, S. Saruwatari, H. Onaga, and H. Morikawa, "Distributed spectrum sensing utilizing heterogeneous wireless devices and measurement equipment," in Proc. DySPAN, 2011, pp. 173-184.

[4] J. J. Lehtomäki, R. Vuohtoniemi, K. Umebayashi, and J.-P. Mäkelä, "Energy detection based estimation of channel occupancy rate with adaptive noise estimation," IEICE Trans. Commun., no. 04, Apr. 2012.

[5] J. Vartiainen, H. Sarvanko, J. Lehtomäki, M. Juntti, and M. Latva-aho, "Spectrum sensing with LAD based methods," in Proc. PIMRC, Athens, Greece, Aug. 2007.

[6] K. Umebayashi, T. Kazmi, Y. Kamiya, Y. Suzuki, and J. Lehtomäki, "Dynamic selection of CWmin in cognitive radio networks for protecting IEEE 802.11 primary users," in Proc. CROWNCOM, Osaka, Japan, Jun. 2011.

[7] A. D. Spaulding and G. H. Hagn, "On the definition and estimation of spectrum occupancy," IEEE Transactions on Electromagnetic Compatibility, vol. EMC-19, no. 3, pp. 269-280, Aug. 1977.

[8] J. J. Lehtomäki, M. Juntti, and H. Saarnisaari, "CFAR strategies for channelized radiometer," IEEE Signal Processing Lett., vol. 12, pp. 1316, 2005. 


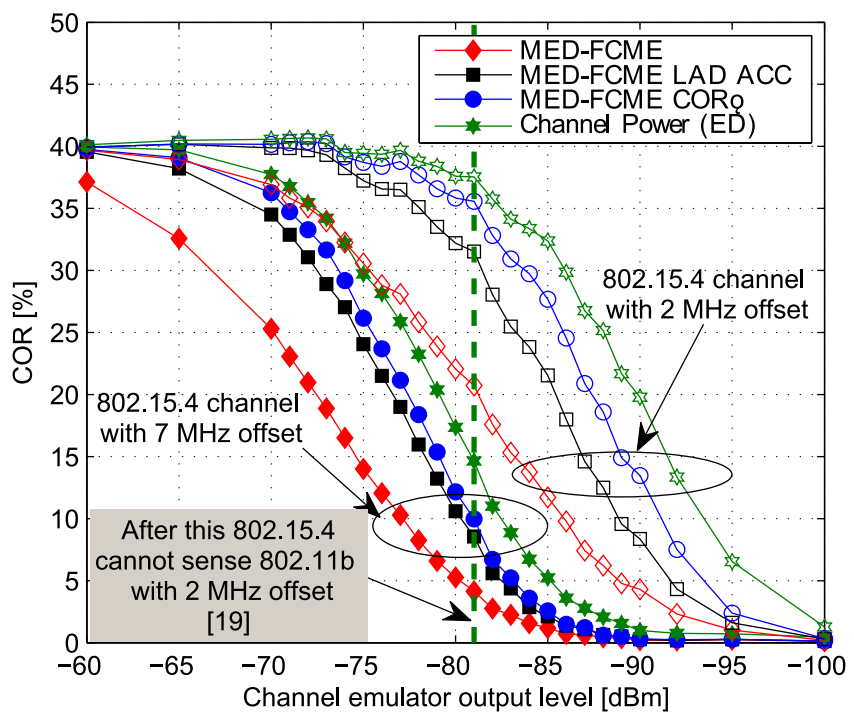

Fig. 5. Measurement results $\left(L_{s}^{k}\right), 802.11 \mathrm{~b}$ signal, ETSI BRAN WLAN channel model A, device speed $5 \mathrm{~km} / \mathrm{h}, r=1$.

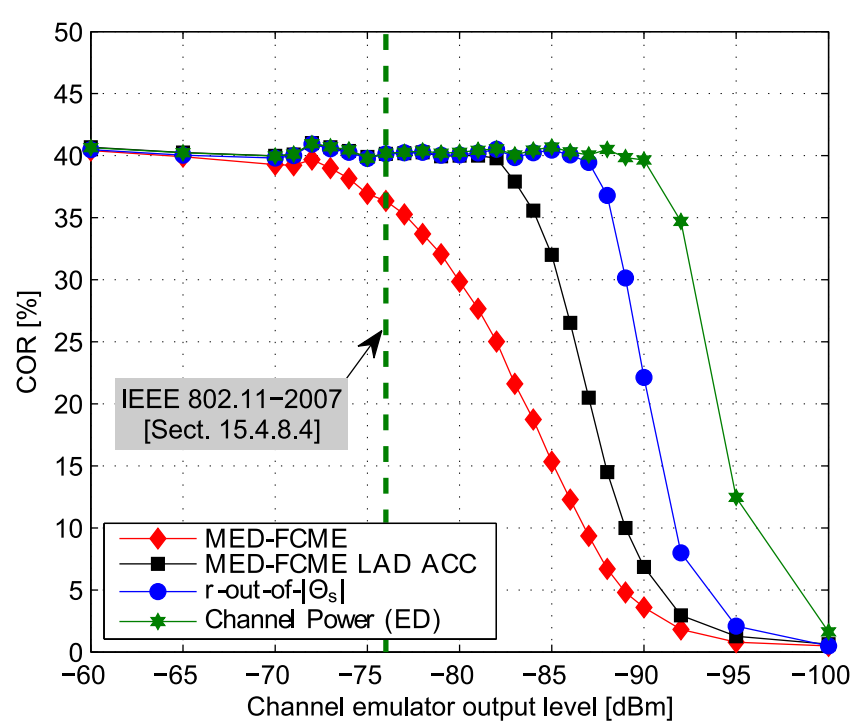

Fig. 6. Measurement results, $802.11 \mathrm{~b}$ signal, AWGN channel, $15 \mathrm{MHz}$ CRS channel, $r=2$.

[9] LAN/MAN Standards Committee, IEEE Std 802.11-2007.

[10] J. Vartiainen, J. J. Lehtomäki, and H. Saarnisaari, "Estimation of signal detection threshold by CME algorithms," in Proc. VTC, Milan, Italy, May 2004.

[11] D. Torrieri, "The radiometer and its practical implementation," in Proc. MILCOM, San Jose, CA, USA, Oct./Nov. 2010.

[12] J. Lehtomäki, J. Vartiainen, R. Vuohtoniemi, and H. Saarnisaari, "Adaptive FCME-based threshold setting for energy detectors," in Proc. CogART, Barcelona, Spain, Oct. 2011.

[13] H. Saarnisaari, P. Henttu, and M. Juntti, "Iterative multidimensional impulse detectors for communications based on the classical diagnostic methods," IEEE Trans. Commun., vol. 53, no. 3, pp. 395-398, Mar. 2005.

[14] J. J. Lehtomäki, J. Vartiainen, M. Juntti, and H. Saarnisaari, "CFAR outlier detection with forward methods," IEEE Trans. Signal Processing,

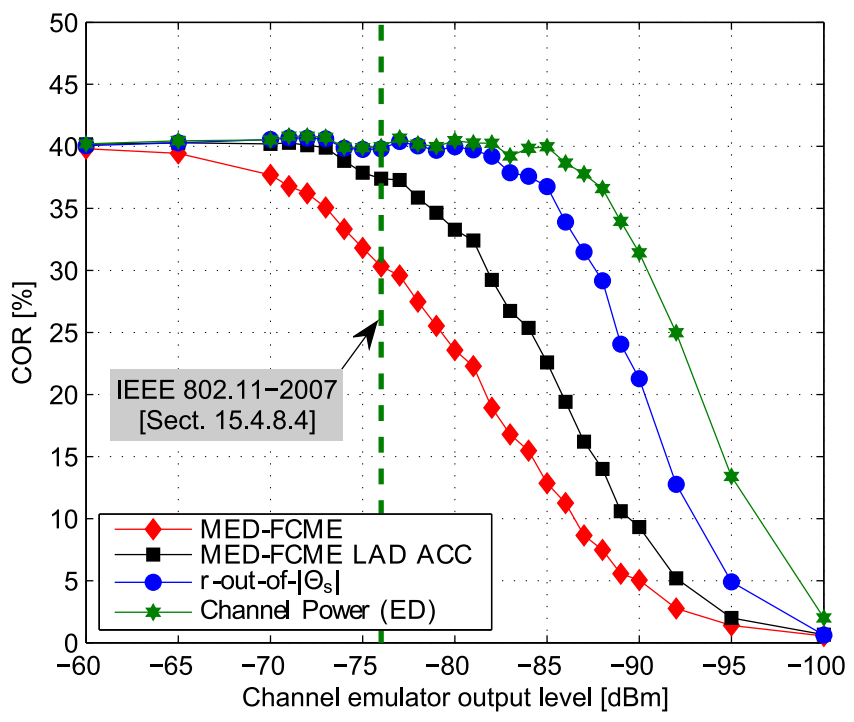

Fig. 7. Measurement results, 802.11b, ETSI BRAN WLAN channel model A, device speed $5 \mathrm{~km} / \mathrm{h}, 15 \mathrm{MHz}$ CRS channel, $r=2$.

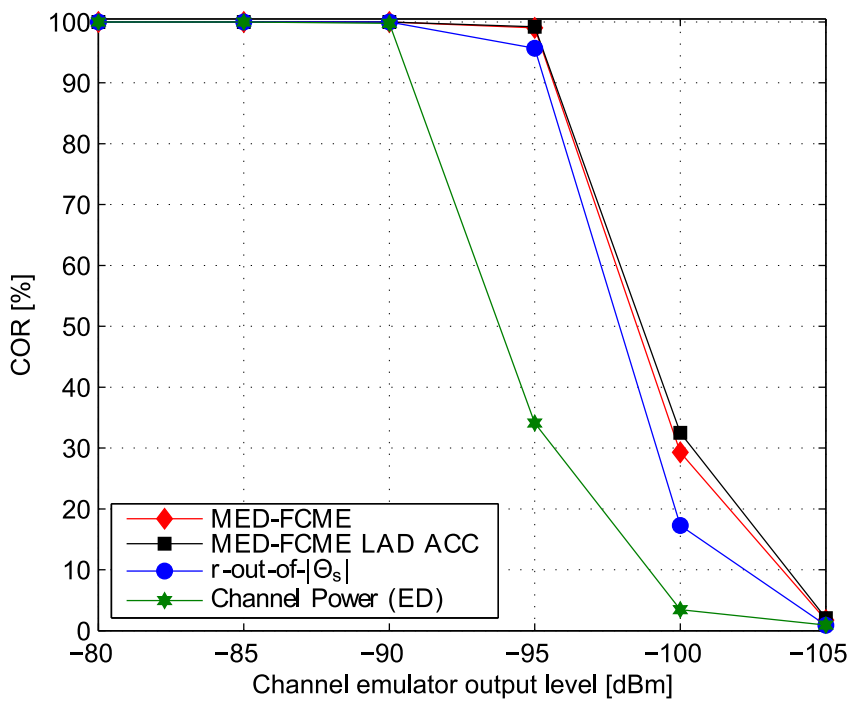

Fig. 8. Measurement results, wideband FM signal, AWGN channel, $15 \mathrm{MHz}$ CRS channel, $r=2$.

vol. 55, no. 9, pp. 4702-4706, Sep. 2007.

[15] J. Vartiainen, J. J. Lehtomäki, and H. Saarnisaari, "Double-threshold based narrowband signal extraction," in Proc. VTC, Stockholm, Sweden, May/June 2005.

[16] B. Levitt, M. Simon, A. Polydoros, and U. Cheng, "Partial-band detection of frequency-hopped signals," in Proc. GLOBECOM, vol. 4, 1993 , pp. 70-76.

[17] S. Geirhofer, L. Tong, and B. M. Sadler, "Dynamic spectrum access in WLAN channels: Empirical model and its stochastic analysis," in Proc. TAPAS, Aug. 2006.

[18] "Channel models for HIPERLAN/2 in different indoor scenarios," ETSI EP BRAN 3ER1085, Mar. 1998.

[19] W. Yuan, X. Wang, J.-P. Linnartz, and I. G. Niemegeers, "Experimental validation of a coexistence model of IEEE 802.15.4 and IEEE 802.11b/g networks," Int. J. Distrib. Sens. Networks, 2010. 